\section{RMD Open}

Rheumatic \& Musculoskeletal Diseases

\title{
Incidence of inpatient venous
} thromboembolism in treated patients with rheumatoid arthritis and the association with switching biologic or targeted synthetic disease-modifying antirheumatic drugs (DMARDs) in the real-world setting

\author{
Huifang Liang, ${ }^{\circ 1}$ Raghava Danwada, ${ }^{1}$ Dianlin Guo, ${ }^{1}$ Jeffrey R Curtis, ${ }^{\circledR} 2$ \\ Ryan D Kilpatrick, ${ }^{1}$ Barbara Hendrickson, ${ }^{3}$ Syed S Islam ${ }^{1}$
}

To cite: Liang H, Danwada R, Guo D, et al. Incidence of inpatient venous thromboembolism in treated patients with rheumatoid arthritis and the association with switching biologic or targeted synthetic diseasemodifying antirheumatic drugs (DMARDs) in the realworld setting. RMD Open 2019;5:e001013. doi:10.1136/ rmdopen-2019-001013

- Additional material is published online only. To view please visit the journal online (http://dx.doi.org/10.1136/ rmdopen-2019-001013).

Received 16 May 2019 Revised 30 August 2019 Accepted 4 September 2019

Check for updates

(c) Author(s) (or their employer(s)) 2019. Re-use permitted under CC BY. Published by BMJ.

For numbered affiliations see end of article.

Correspondence to Dr Huifang Liang; huifang.liang@abbvie.com

\section{ABSTRACT}

Objectives To assess incidence rates (IRs) of VTE in patients with rheumatoid arthritis (RA) on different DMARDs and DMARD switchers.

Methods Adults with RA on a DMARD between 2007 and 2017 were studied in a US claims database. Conventional synthetic DMARD (csDMARD) users, first biologic/targeted synthetic DMARD (b/tsDMARD) users and b/tsDMARD switchers (from a b/tsDMARD to another b/tsDMARD) were followed for inpatient VTE (pulmonary embolism (PE)/deep vein thrombosis (DVT)). Crude and adjusted IR and 95\% Cls of VTE were estimated. HRs for VTE were estimated via Cox regression. VTE risk was also evaluated by number of switches between b/tsDMARDs and in patients without a VTE history.

Results The age and sex standardised IR $(95 \% \mathrm{Cl})$ of VTE (per 100 person-years) was 0.86 (0.70 to 1.03$), 0.60$ (0.52 to 0.68 ) and 0.58 (0.51 to 0.65 ) for b/tsDMARD switchers, first b/tsDMARD users and csDMARD users, respectively. After adjustment, b/tsDMARD switchers had an increased risk of VTE, compared with csDMARD users, $\mathrm{HR}_{\text {adj }}(95 \% \mathrm{Cl})$ being 1.36 (1.16 to 1.58), 1.36 (1.13 to 1.63) and 1.47 (1.18 to 1.83) for VTE, DVT and PE, respectively. Compared with first b/tsDMARD users, the $\mathrm{HR}_{\text {adi }}(95 \% \mathrm{Cl})$ for VTE was 1.35 (1.15 to 1.60) for first b/tsDMARD switchers and 1.48 (1.19 to 1.85) for second b/tsDMARD switchers.

Conclusions In RA, b/tsDMARD switchers have a higher VTE risk compared with csDMARD users and first b/ tsDMARD users. Switching b/tsDMARDs may be a proxy for higher disease severity or poorly controlled RA and an important confounder to consider in obtaining unbiased estimates of VTE risk in observational RA safety studies.

\section{INTRODUCTION}

Venous thromboembolism (VTE), including deep vein thrombosis (DVT) and pulmonary

\section{Key messages}

What is already known about this subject?

- The risk of venous thromboembolism (VTE) is increased in patients with rheumatoid arthritis (RA).

What does this study add?

- After controlling for multiple risk factors, patients with RA who switch biologic/targeted synthetic disease-modifying antirheumatic drugs (b/tsDMARDs) have a higher VTE risk compared with conventional synthetic DMARD users and first b/tsDMARD users. This risk was even higher in the subgroup who switched b/tsDMARDs twice.

- Switching may be a proxy for higher disease severity or poorly controlled RA and an important confounder to consider in obtaining unbiased estimates of VTE risk in observational RA safety studies.

How might this impact on clinical practice?

- Drug safety studies may want to consider treatment switching as a potential proxy for unmeasured clinical RA disease activity and severity which may confound associations with important clinical outcomes such as VTE.

embolism (PE), is the third most common cardiovascular disease after myocardial infarction (MI) and stroke. ${ }^{1}$ In the general population, the risk of VTE is approximately $0.1-0.4$ per 100 person-years. ${ }^{1-3}$ Recent studies consistently showed that rheumatoid arthritis (RA) is associated with a 1.5-fold to 1.6-fold increased risk of VTE compared with the general population. ${ }^{3-8}$ The increased risk in RA is believed to be attributable in part to 
proinflammatory cytokine production, oxidative stress and endothelial dysfunction. ${ }^{9}{ }^{10}$ Systemic inflammation modulates thrombotic responses by suppressing fibrinolysis, upregulating procoagulant and downregulating anticoagulants. ${ }^{11}$

Patients with RA may be initially treated with conventional synthetic disease-modifying antirheumatic drugs (csDMARDs) ${ }^{12}$ Patients with moderate to severe RA who do not respond to csDMARDs may switch to biologic or targeted synthetic DMARDs (b/tsDMARDs), which may be administered with or without csDMARDs. The switch from csDMARD to b/tsDMARDs or from one b/ tsDMARD to another $\mathrm{b} / \mathrm{tsDMARD}$ may indicate inability to control systemic inflammation, which may predispose these patients with RA to a higher risk of VTE. Patients who are bDMARD users have been found to have more severe RA (assessed by treatment) than patients treated with csDMARDs. ${ }^{13} 14$

In addition to RA, higher risk of VTE has also been associated with increasing age ${ }^{1516}$ male sex, ${ }^{17-19}$ African-American race, ${ }^{16}$ alcohol abuse, ${ }^{20}$ drug abuse, smoking, ${ }^{21}{ }^{22}$ a history of prior VTE, ${ }^{23}$ cardiovascular risk factors (obesity, hypertension, diabetes mellitus, hyperlipidemia, congestive heart failure (CHF), MI and stroke), ${ }^{24}$ hospitalisation and recent surgery, ${ }^{25}$ chronic kidney disease (CKD), ${ }^{26}$ any malignancy, ${ }^{27}$ serious infections, ${ }^{28}{ }^{29}$ chronic obstructive pulmonary disease (COPD), ${ }^{15}{ }^{30}$ asthma, ${ }^{31}{ }^{32}$ viral hepatitis, ${ }^{33}$ diverticular disease, ${ }^{34}$ gastroduodenal ulcer, ${ }^{35}$ inflammatory bowel disease, ${ }^{36}$ depression, and major knee and hip surgeries. ${ }^{36} 37$

Limited data are available on risk of VTE in DMARD users. Kim et al reported the incidence rates (IRs) of hospitalised VTE in CsDMARD initiators and bDMARD initiators. ${ }^{6}$ Weinblatt et al recently reported the IR of VTE in patients with RA treated with baricitinib, a tsDMARD. ${ }^{38}$ Beyond reporting only overall rates of VTE, $\mathrm{b} / \mathrm{tsDMARD}$ switching in real-world data that may be proxies for higher RA disease severity and more refractory patients who may be at greater risk of VTE are important to identify. This information would potentially increase the validity of future observational drug safety studies. To this end, DMARD-treated patients in this analysis were characterised by line of therapy and further, by whether and how many times they switched from $\mathrm{a} b /$ tsDMARD to another $\mathrm{b} /$ tsDMARD. We evaluated whether VTE rates could be increased according to line of RA therapy and b/tsDMARD switching, which may be a useful proxy for higher disease severity or refractory RA.

\section{METHODS}

\section{Study design and data source}

A cohort study was conducted using the Optum Clinformatics Data Mart, a US claims database containing anonymised longitudinal data for patients insured with the United HealthCare plans since 2000.

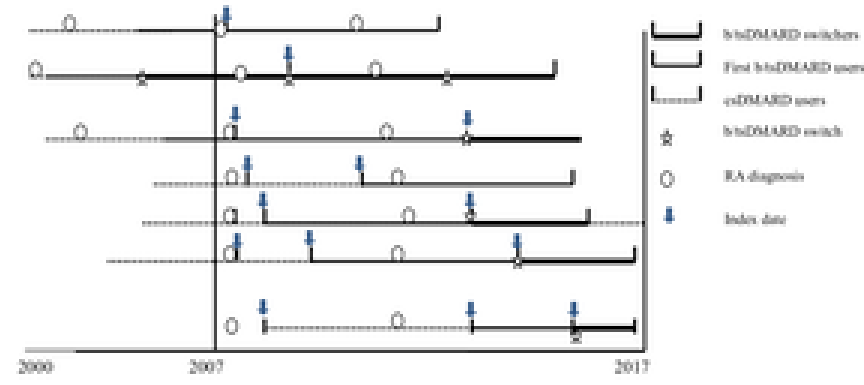

Figure 1 Identification of biologic/targeted synthetic disease-modifying antirheumatic drug (b/tsDMARD) switchers, first b/tsDMARD users and conventional synthetic DMARD users during the study period. Note: the first treatment after the diagnosis of rheumatoid arthritis that met the definition of treatment group and during the study period (2007-2017) was used to define the treatment group.

\section{Study population}

Adults were eligible for the study if they had at least two diagnoses of RA (see online supplemental data) from medical claims $\geq 7$ days apart in 2007-2017, received at least one systemic DMARD after the first RA diagnosis in 20072017 and had at least 1year of continuous health plan enrolment before the index DMARD treatment, with a gap of $\leq 45$ days allowed. The index date was defined as the first date of DMARD treatment after the first RA diagnosis in the study period. No exclusion criteria were applied.

\section{DMARD treatment}

Figure 1 presents a diagram to identify three cohorts; some patients might be in multiple cohorts. During the study period, those who discontinued one or more previous b/tsDMARDs (see online supplemental data for generic names) and switched to another $\mathrm{b} / \mathrm{tsDMARD}$ were defined as b/tsDMARD switchers. Switching from intravenous administration to subcutaneous administration of the same drug or vice versa was not considered a switch. First b/tsDMARD users were those on their first observed $\mathrm{b} /$ tsDMARD therapy. Both b/tsDMARD switchers and first b/tsDMARD users may be treated with csDMARD previously and concomitantly. csDMARD users were those who received csDMARD but had not received any $\mathrm{b}$ /tsDMARDs. Since our objective was not to evaluate the effect of RA therapy (together or individually) but to assess the ability of switching among $\mathrm{b} / \mathrm{tsDMARDs}$ to serve as a proxy for uncontrolled disease activity on VTE risk, individual drugs were not assessed in the primary analysis. Others have discussed or explored differences in VTE rates in those treated with tsDMARDs. ${ }^{3940}$ Therefore, as a secondary analysis, the incidence rate of VTE was also explored by drug group (TNF- $\alpha$ inhibitors, non-TNF- $\alpha$ biologics, tsDMARD tofacitinib and biosimilars).

\section{Outcomes and covariates}

The VTE outcome was defined as the first hospitalised DVT or PE during follow-up, while when analysed separately, the earliest hospitalised DVT during follow-up and the earliest hospitalised PE during follow-up. ${ }^{64142}$ Data 
on patient characteristics (age, sex, race, calendar year and geographical region), behavioural factors (smoking, alcohol/drug abuse), hospitalisation within 1 year before index date, cardiovascular risk factors, comorbidities/ medical histories, history of dispensed non-steroidal anti-inflammatory drugs (NSAIDs, including Cox-2 inhibitors), corticosteroids and anticoagulants any time before the cohort entry were also included as covariates. For medical history or comorbidities, we required two outpatient diagnoses on different dates or one inpatient diagnosis. Hospitalisation was required for serious infections. Although some patients may have contributed to different treatment cohorts, the time windows for their baseline characteristics and outcome ascertainment were different.

\section{STATISTICAL ANALYSES}

Descriptive statistics were generated for baseline patient characteristics. A standardised difference with an absolute value of $\geq 0.1$ was used to indicate meaningful difference between groups at baseline. ${ }^{43}$ The follow-up started from the day after the index date to the earliest of an outcome event, disenrolment from the healthcare plan, treatment discontinuation, death while hospitalised or 30 September 2017. The treatment time window was defined from the index date through the last drug dose plus an exposure window of 90 days for b/tsDMARDs ${ }^{44}$ and 37 days for csDMARD, based on a 30-day discontinuation gap for methotrexate ${ }^{45}$ plus 7 days of prefilling a prescription. The on-treatment approach was used to assess the IR of VTE with justifications (online supplemental data). The 1-year cumulative incidence was estimated using the number of inpatient VTE cases within 1 year after the index date divided by the number of patients. The number needed to harm $(\mathrm{NNH})$ was calculated by dividing 1 by the absolute risk increase for $\mathrm{b} /$ tsDMARD switchers and first b/tsDMARD users, respectively, with csDMARD users as the reference group.

For csDMARD users, the follow-up ended at the time of initiating a b/tsDMARD; for first b/tsDMARD users, the follow-up ended at the time of switching to a second $\mathrm{b} / \mathrm{tsDMARD}$. The follow-up of $\mathrm{b} / \mathrm{tsDMARD}$ switchers continued when they switched to other b/tsDMARDS. In the main analysis, no further distinction was made among $\mathrm{b} / \mathrm{tsDMARD}$ switchers (ie, all b/tsDMARD switchers were represented as a single exposure group). However, in a sensitivity analysis, b/tsDMARD switchers were further classified into 'first' b/tsDMARD switchers (of note, some 'first' b/tsDMARD switchers may have switched before the index date) and 'second' b/tsDMARD switchers after the index date, with the time of switch to a different b/ tsDMARD treated as a censoring event for VTE for both cohorts. Eligibility criteria were re-assessed and covariates were updated for 'second' b/tsDMARD switchers based on the second switch date. Due to significant reduction in number of patients, risks beyond the 'second' b/ tsDMARD switchers were not assessed.

The crude IR of first hospitalised VTE, DVT and PE occurring after the start of follow-up and $95 \% \mathrm{CIs}^{46}$ were estimated by treatment groups and selected patient characteristics. Standardised IRs were calculated for three treatment groups, and subgroup of drugs among biologic users, applying age and sex-specific IRs to population age and sex distribution from US Census 2010 in a direct method, ${ }^{47}$ with $95 \%$ CI estimated using normal approximation. ${ }^{48}$

Cox proportional-hazards models were used to estimate the effect (HRs) of treatment on the risk of VTE outcomes, with primary model adjusted for demographics. Additional variable selection was based on clinical knowledge and F statistic from Cox models. Data management and statistical analyses were performed using SAS V.9.4 (SAS Institute, Cary, North Carolina, USA). Institutional review board review is exempt for this study as data were de-identified.

\section{RESULTS}

Patient disposition is presented in figure 2. Table 1 presents patient characteristics for b/tsDMARD switchers, first b/tsDMARD users and csDMARD users. Each cohort had an average enrolment period of 4 years or more before the index date. A total of 14823 (16.0\% of 92 509) csDMARD users became first b/tsDMARD users, and 9757 (25.7\% of 37 993) first b/tsDMARD users became b/tsDMARD switchers (data not shown). $\mathrm{b} / \mathrm{tsDMARD}$ users were on average 5.5 years younger than csDMARD users. The most common disease history or comorbidities in the study population were hypertension $(>40 \%)$ and hyperlipidemia $(\sim 40 \%)$, followed by diabetes mellitus $(>15 \%)$, serious infections $(>10 \%)$, CKD, COPD, asthma, depression $(\sim 10 \%)$, inflammatory bowel disease, malignancy and $\mathrm{MI} /$ stroke $(<10 \%)$. Compared with csDMARD users, first b/tsDMARD users had a lower proportion of hypertension, hyperlipidemia, diabetes mellitus, CHF, atrial fibrillation or flutter, and $\mathrm{MI} /$ stroke. b/tsDMARD switchers had a higher proportion of patients with a history of serious infections, major knee and hip surgeries, and corticosteroid use than first $\mathrm{b} /$ tsDMARD users and csDMARD users. No differences were identified between groups for behavioural risk factors and inpatient hospital stays (online supplemental table S1).

Crude IRs of VTE, DVT and PE stratified by treatment groups and patient characteristics are presented in table 2. The overall crude IR $(95 \% \mathrm{CI})$ of VTE was 0.79 (0.75 to 0.83$)$ per 100 patient-years(PY). Among those who did not use anticoagulants in 1 year before index date, the IR of VTE was 0.62 (0.58 to 0.66 ) per 100 patientyears. The stratified analysis demonstrated that increased risk of VTE was associated with b/tsDMARD switching, male sex, increasing age, white and African-American races, behaviour factors, medical histories and history of 


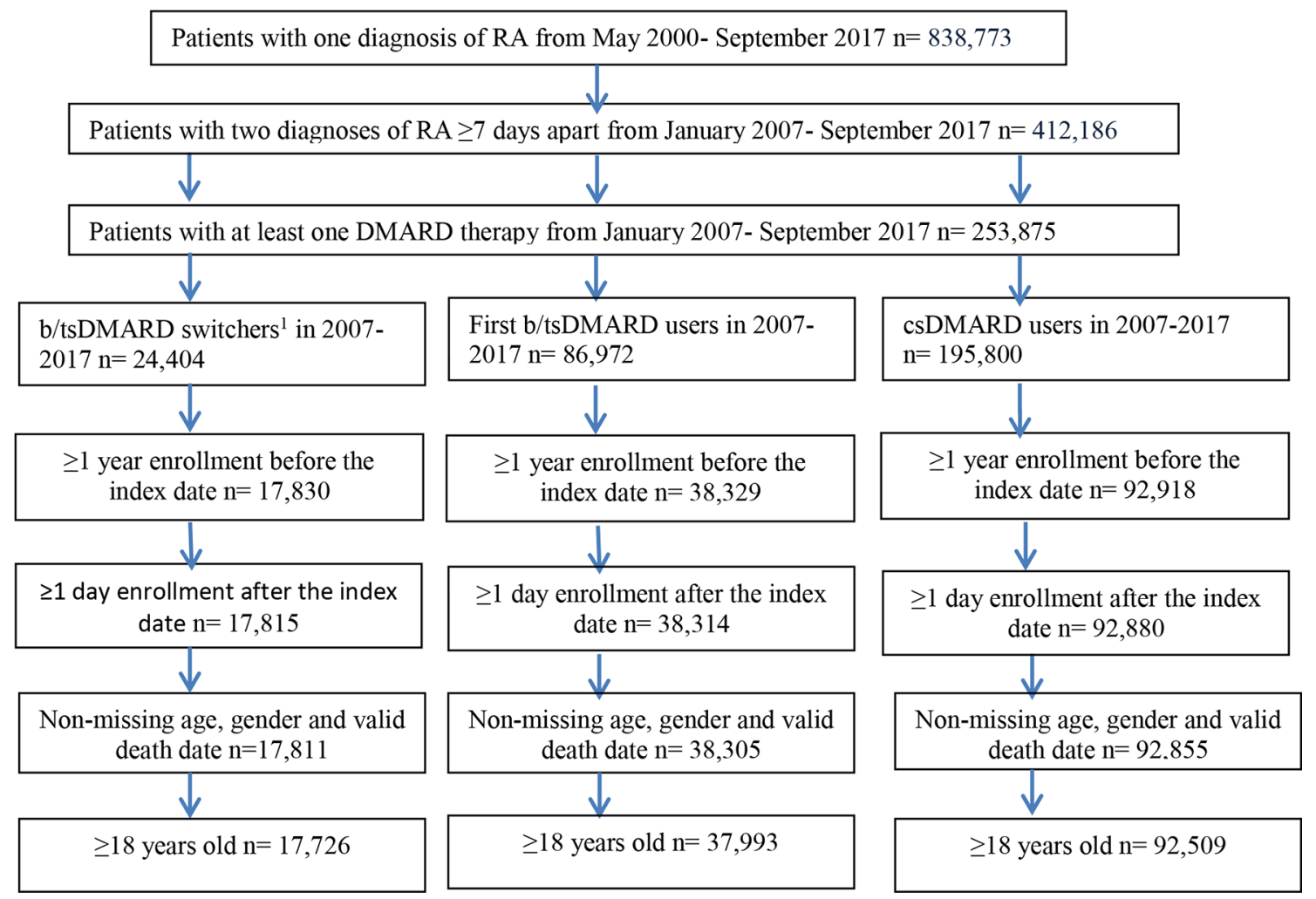

Figure 2 Patient attrition in patients with rheumatoid arthritis in the Optum Cliniformatics Data Mart from May 2000 through September 2017.

medication use including dispensed anticoagulant and corticosteroid. NSAID use was not associated with an increased risk of VTE (online supplemental table S2).

Using the US Census population estimates on 1 July 2010, the age-standardised and sex-standardised IR of VTE was 0.86 (95\% CI 0.70 to 1.03 ) per 100 patientyears in RA b/tsDMARD switchers, higher than that in the first b/tsDMARD users $(0.60,95 \%$ CI 0.52 to 0.68$)$ and that in the csDMARD users $(0.58,95 \%$ CI 0.51 to $0.65)$. When we stratified by history of MI or stroke, the age-standardised and sex-standardised IR of VTE among those without a history of MI or stroke was 0.79 (0.62 to 0.97$)$ in RA b/tsDMARD switchers, 0.53 (0.45 to 0.60$)$ in the first b/tsDMARD users and $0.51(0.43$ to 0.58 ) in the csDMARD users. The standardised IR of VTE in first $\mathrm{b} / \mathrm{tsDMARD}$ users was not different from that in csDMARD users in overall and the stratified analysis. Similar patterns were observed for DVT and PE (table 3). The majority of patients received TNF- $\alpha$ inhibitors as both an initial and subsequent (postswitch) therapy. When subgroups of $b /$ tsDMARDs were explored, however, the age-standardised and sex-standardised VTE rates were comparable between TNF- $\alpha$ inhibitors and non-TNF- $\alpha$ biologics for both switchers and first users. Compared with TNF- $\alpha$ inhibitors and non-TNF- $\alpha$ biologics, the age-standardised and sex-standardised rate of VTE for tsDMARD was similar among first users but numerically lower among switchers, which might not be a reliable finding due to small numbers of tofacitinib users (the only tsDMARD evaluable in this study) (online supplemental table S3). The annual incidence for $\mathrm{b} / \mathrm{tsDMARD}$ switchers, first $\mathrm{b} / \mathrm{tsDMARD}$ users and csDMARD users was $0.75 \%(133 / 17726), 0.57 \%$ $(216 / 37993)$ and $0.49 \%(457 / 92509)$. Compared with csDMARD users, the NNH was 391 for b/tsDMARD switchers and 1342 for first b/tsDMARD users.

The adjusted HR $\left(\mathrm{HR}_{\text {adj }}\right)$ and 95\% CI for VTE from multivariate Cox regression are presented in figure 3. Model 1 is our primary model. When age, sex and race were adjusted (model 1), the risk of VTE among b/ tsDMARD switchers was about $50 \%$ higher than that of csDMARD users and $35 \%$ higher than first $\mathrm{b} /$ tsDMARD users. After adjusting for additional variables including serious infections, hypertension, hospitalisations, COPD, CKD, MI or stroke, the $\mathrm{HR}_{\text {adj }}$ barely changed (from 1.39 in model 2 to 1.36 in model 3 in online supplemental table S4). The HRs were slightly attenuated with more risk factors adjusted from model 1 to model 2 to model 3 , which suggested that the increased risk of VTE among $\mathrm{b} /$ tsDMARD switchers could be explained by differences in the medical histories. No significant difference in risk of VTE was identified between first b/tsDMARD users and csDMARDs users in any of these models. Similar patterns were observed for DVT and PE (online supplemental table S4). Other identified risk factors for VTE included history of VTE, anticoagulant use, serious infections, hypertension, hospitalisations, COPD, CKD and $\mathrm{MI} /$ stroke. The findings were consistent among those without a history of VTE, anticoagulant use and malignancy (online supplemental table S5). 
¿

类

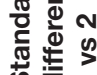

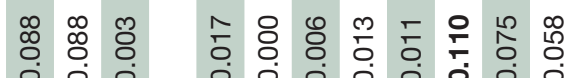

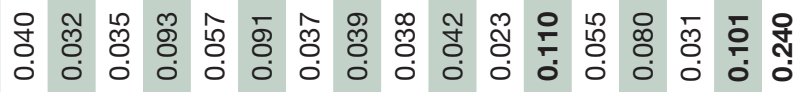

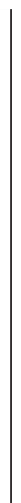

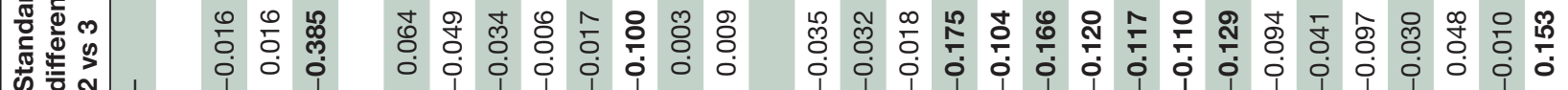

후

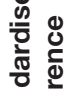

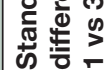

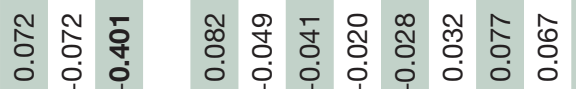
융ำ

尊

$\sum^{5}$ o 


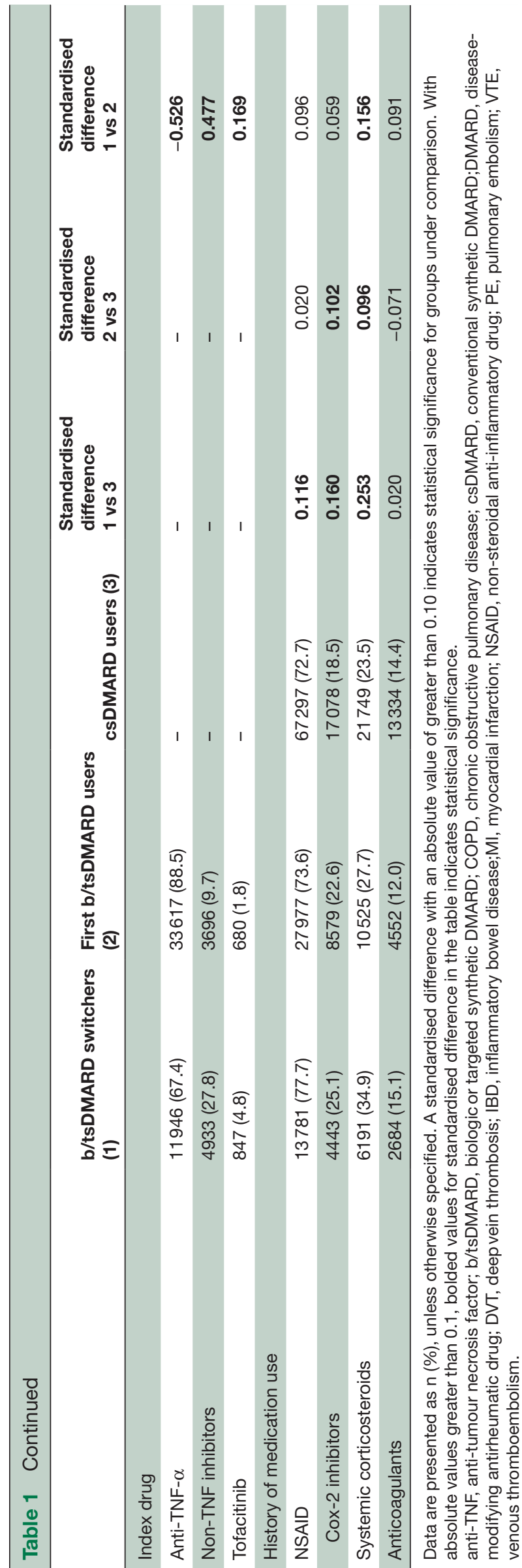

In sensitivity analyses, the crude IR (95\% CI) of VTE was 0.96 (0.78 to 1.16 ) per $100 \mathrm{PY}$ for 'second' b/tsDMARD switchers and 0.87 ( 0.74 to 1.02 ) per 100 PY for 'first' $\mathrm{b} / \mathrm{tsDMARD}$ switchers (online supplemental table S6). Compared with first b/tsDMARD users, controlling for age and sex and race, the HR (95\% CI) for VTE was 1.48 (1.19 to 1.85) for 'second' b/tsDMARD switchers and 1.35 (1.15 to 1.60 ) for 'first' b/tsDMARD switchers (online supplemental table $\mathrm{S} 7$ ); in patients without a history of VTE, the HR remained significantly higher (second switchers HR 1.44, 95\% CI 1.13 to 1.83; first switchers $1.29,95 \%$ CI 1.07 to 1.56 ) (online supplemental table S8). The risk of DVT and PE followed similar patterns in all analyses.

\section{DISCUSSION}

In this analysis, we found that VTE risk was increased with advancing line of RA therapy, which may reflect line of therapy being a proxy for increasing disease severity and inadequate control of RA disease activity. With the increasing availability of $\mathrm{b} / \mathrm{tsDMARD}$ options for $\mathrm{RA}$, our finding that $\mathrm{b} / \mathrm{tsDMARD}$ switching was associated with significantly increased VTE risk is particularly important for future observational studies of drug safety using administrative healthcare databases when standardised RA disease activity measures are unavailable. Specifically, we found an increased IR of VTE among patients with RA who switched from b/tsDMARDs to alternative b/tsDMARDs, when compared with either csDMARD users ( $\mathrm{HR}_{\text {adj }} 1.36,95 \%$ CI 1.16 to 1.58$)$ or first $\mathrm{b} / \mathrm{tsDMARD}$ users $\left(\mathrm{HR}_{\mathrm{adj}} 1.20,95 \%\right.$ CI 1.02 to 1.42$)$. The increased risk was more pronounced in the second-time $\mathrm{b} / \mathrm{tsDMARD}$ switchers than in the first-time $\mathrm{b} / \mathrm{tsDMARD}$ switchers. This study also confirmed several independent risk factors of VTE in RA patient population.

Our findings are consistent with the current literature on RA. ${ }^{56}$ Although the overall crude IR of VTE in this study $(0.79,95 \%$ CI 0.75 to 0.83$)$ was somewhat higher than what was reported by Kim et al $(0.61,95 \%$ CI 0.54 to 0.69$),{ }^{5}$ this discrepancy is likely due to differences in study population and methodology. Kim et alstudied a RA cohort from different data sources in an earlier calendar time period and excluded patients with claims for DVT or PE, or anticoagulants dispensing in the 12-month period before index date. Among similarly selected patients without anticoagulant use within 1 year before index date, the IR of VTE in our study $(0.62,95 \%$ CI 0.58 to 0.66 per $100 \mathrm{PY}$ ) was consistent with theirs. ${ }^{5}$

Unlike other reports, this study estimated the IR of VTE in the subgroup of patients with RA who switched from one $\mathrm{b} /$ tsDMARD to another. Switching from one $\mathrm{b} / \mathrm{tsDMARD}$ to another $\mathrm{b} / \mathrm{tsDMARD}$ may identify patients with insufficient response or intolerance to b/ tsDMARD therapy in a real-world setting. Patients who are BDMARD users have been found to have more severe RA than patients treated with csDMARDs. ${ }^{13}{ }^{14}$ In this study, despite on average being younger and with a 


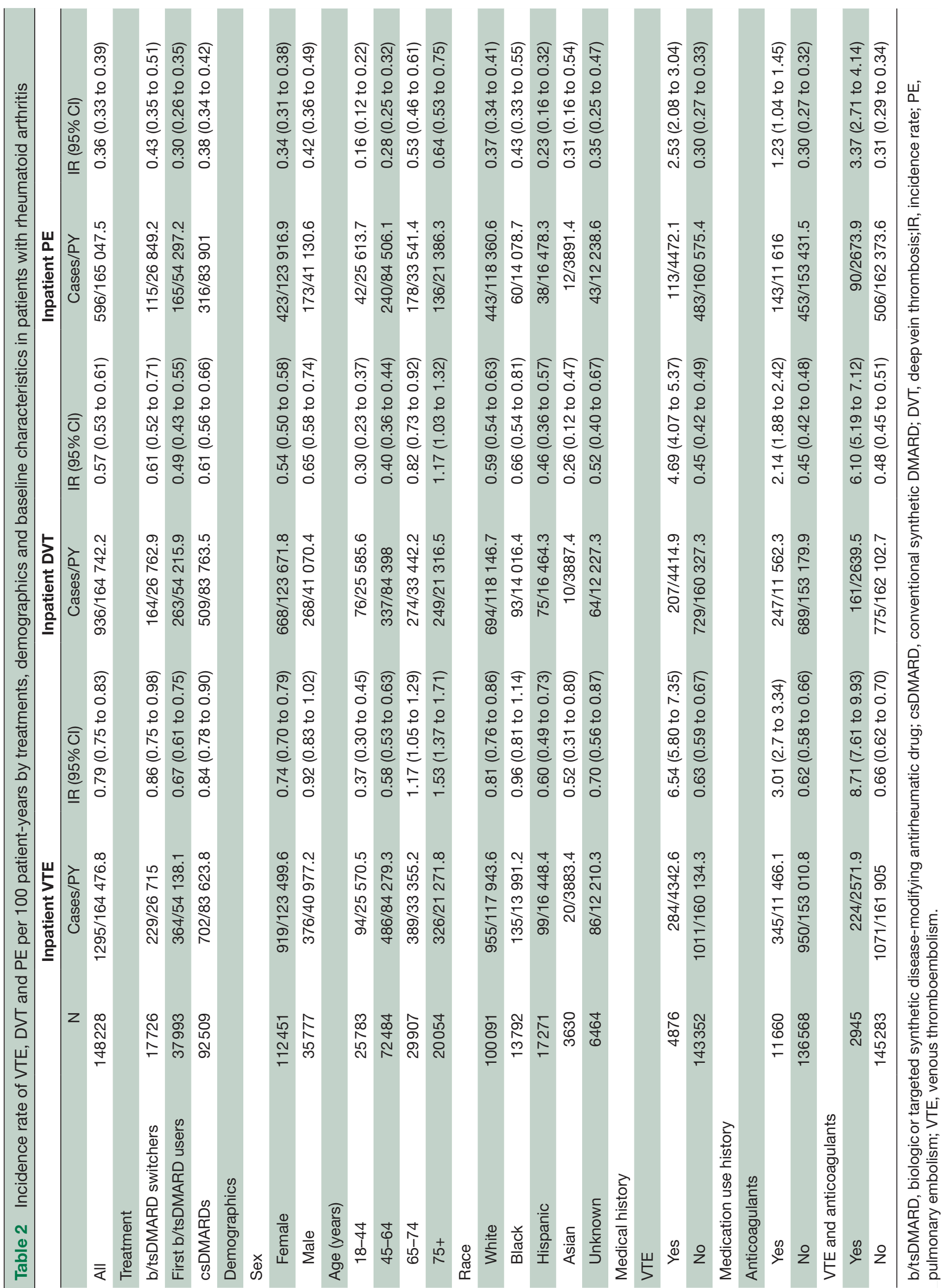


Table 3 Age and sex-standardised incidence rates and 95\% Cls in b/tsDMARD switchers, first b/tsDMARD users and csDMARD users

\begin{tabular}{lllr}
\hline & b/tsDMARD switchers & First b/tsDMARD users & csDMARD users \\
\hline VTE & $0.86(0.70$ to 1.03$)$ & $0.60(0.52$ to 0.68$)$ & $0.58(0.51$ to 0.65$)$ \\
DVT & $0.57(0.44$ to 0.70$)$ & $0.45(0.38$ to 0.52$)$ & $0.42(0.36$ to 0.48$)$ \\
PE & $0.48(0.34$ to 0.61$)$ & $0.27(0.21$ to 0.32$)$ & $0.26(0.22$ to 0.31$)$ \\
\hline
\end{tabular}

Age and sex-standardised incidence rate was calculated by applying age and sex-specific incidence rates (ie, 18-44, 45-64, 65-74 and 75+ for men and women) to population age and sex distribution from US Census 2010 in a direct method, ${ }^{47}$ with $95 \%$ Cls estimated using normal approximation. ${ }^{48}$

b/tsDMARD, biologic or targeted synthetic disease-modifying antirheumatic drug; csDMARD, conventional synthetic DMARD; DVT, deep vein thrombosis; PE, pulmonary embolism; VTE, venous thromboembolism.

similar pre-enrolment period, b/tsDMARD switchers had a higher proportion of serious infections, major knee and hip surgeries, and co-medication use indicating on average they had more severe RA. Since anti-TNF therapy appeared to be associated with reduced risk of VTE, ${ }^{49}$ the increased risk among $\mathrm{b} / \mathrm{tsDMARD}$ switchers in this study could be due to continued inflammatory process ${ }^{50}$ (persistent disease severity) despite b/tsDMARD treatment and factors associated with treatment option, rather than treatments themselves. Although there was some attenuation in HR of VTE for b/tsDMARD switchers when additional risk factors reflecting comorbidities and disease severity were adjusted, even with adjustment of additional factors, b/tsDMARD switching remained associated with a significantly higher risk of VTE compared with first $b$ / tsDMARD users or csDMARD users. As measures of RA disease activity or severity, such as Disease Activity Score or clinical disease activity index, were unavailable in claims data, switching b/tsDMARDs may be a proxy for higher disease severity or more poorly controlled RA.

We acknowledge that in a real-world setting, patients may switch RA treatments due to reasons such as insurance coverage or adverse events. However, the effect of such switching would be to underestimate, not overestimate, the differences in disease severity measures between the groups. Nevertheless, the increased risk in the second-time $\mathrm{b} / \mathrm{tsDMARD}$ switchers, followed by the first-time $b / t s D M A R D$ switchers and the first $b / t s D M A R D$ users, demonstrated the utility of including switching b/ tsDMARDs as a disease severity indicator.

In addition, this study confirmed several independent risk factors for VTE in patients with RA. Although risk factors such as increasing age, male sex and African-American race have been reported in general population, to the best of our knowledge, this is among the few US studies to report VTE risk factors that are relatively common in RA populations: history of VTE, history

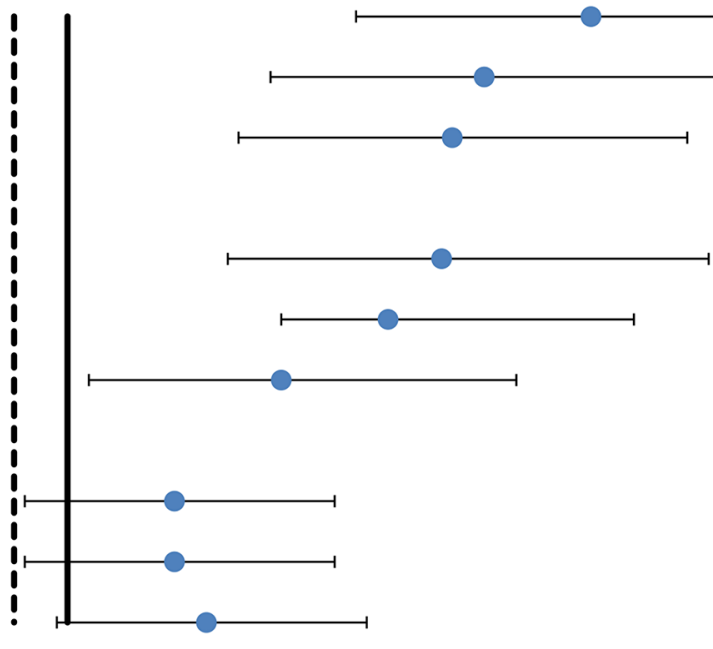

$\begin{array}{lll} & 1.35(1.15,1.60) & 1 \\ \text { b/tsDMARD switchers } & 1.30(1.20,1.53) & 2 \\ \text { vs csDMARD users } & 1.20(1.02,1.42) & 3 \\ & & \\ & & \\ \text { first b/tsDMARD users } & 1.10(0.96,1.25) & 1 \\ \text { vs csDMARD users } & 1.10(0.96,1.25) & 2 \\ & 1.13(0.99,1.28) & 3\end{array}$

0.8

1
1.2
1.4

Hazard ratio and $95 \%$ confidence intervals
HR $(95 \%$ CI) Model

$1.49(1.27,1.73) \quad 1$

$1.39(1.19,1.62) \quad 2$

$1.36(1.16,1.58) \quad 3$

Figure $3 \mathrm{HRs}$ and 95\% Cls from multivariate Cox proportional-hazards model for venous thromboembolism (VTE) in patients with rheumatoid arthritis on biologic or non-biologic therapy. Note: model 1 adjusted for age, sex and race; model 2 adjusted for variables in model 1, plus history of VTE, and anticoagulant use at baseline; model 3 adjusted for variables in model 2, plus serious infections, hypertension, hospitalisation, chronic obstructive pulmonary disease, chronic kidney disease and myocardial infarction/stroke. 
of anticoagulant use, serious infections, hypertension, hospitalisation, COPD, CKD and MI/stroke.

This study is subject to several limitations. First, the VTE outcome was identified based on ICD diagnosis on inpatient medical claims without further chart review. However, this method has been validated to have a positive predictive value $\geq 74 \% .^{51}$ Further, misclassification of the outcome is likely to be non-differential with respect to the treatment groups, thereby leading to bias towards the null. Second, we used b/tsDMARD switchers and number of switches (line of therapy) as our proxy for inadequate response to treatment and more severe RA. Misclassification of the number of previous cs/b/ tsDMARDs used was possible due to availability of data (ie, left-censoring of events prior to enrolment in the health plan), which would have the conservative effect of biasing our results towards the null. It will be important to correctly classify patients' biologic treatment history in future comparative safety analyses of VTE. Third, although several baseline medical conditions and medications were identified as independent risk factors in our analysis, RA disease activity or severity was unavailable in the database. Analysing RA registry or EHR data where disease activity and severity are available may help further understand the difference in risk of VTE among patients with different treatment experience. Further, this study identified inpatient VTE. Patients at low risk (eg, without CHF or severe liver impairment) for mortality may be treated as outpatients. ${ }^{52}$ However, outpatient VTE is less reliable and has not been used by comparative studies in RA. ${ }^{56}$ Although the incidence of VTE was underestimated, we used inpatient VTE to improve comparability between treatment groups. Fourth, stepwise regression was used in our analysis, the limitations of which have been described. ${ }^{53}$ Finally, this study focused on US patients treated with csDMARD or b/tsDMARD, and our study findings may not be generalisable to patients with RA in a different country.

In summary, this large US study provides incidence rates of VTE in patients with RA according to prior switching as a proxy for more severe disease in a realworld setting. Patients with RA with more extensive treatment experience, especially b/tsDAMRD switchers, have a higher risk of VTE compared with those treated with csDMARD and first b/tsDAMRD users. The heightened risk of VTE in b/tsDMARD switchers was somewhat attenuated when factors that reflect co-medications and comorbidities were controlled, yet remained significantly elevated, suggesting switching to an alternative $\mathrm{b} / \mathrm{tsDMARD}$ may be a useful proxy for higher disease severity or poorly controlled RA in patients with other constellation of chronic conditions and risk factors. In addition to line of therapy, b/tsDMARD switching and the number of switches may be an important and independent risk factor to include moving forward in observational RA drug safety studies, particularly those using secondary data sources that lack standardised disease activity measures.
Author affiliations

${ }^{1}$ Global Epidemiology, Pharmacovigilance and Patient Safety, AbbVie Inc, North Chicago, Illinois, USA

${ }^{2}$ Division of Clinical Immunology and Rheumatology, University of Alabama at Birmingham, Birmingham, Alabama, USA

${ }^{3}$ Pharmacovigilance and Patient Safety, AbbVie Inc, North Chicago, Illinois, USA

Acknowledgements The design, study conduct and financial support for the study were provided by AbbVie. AbbVie participated in the interpretation of data, review and approval of the publication. The authors wish to thank Drs Mondira Bhattacharya, Jerzy Tyczynski and Dongmu Zhang for providing valuable comments on the study protocol and Brian Waterhouse for his input in statistical modelling.

Contributors $\mathrm{HL}$ contributed to study concept and design, data acquisition, data analysis, data interpretation, drafting of the initial manuscript, critical revision for important intellectual content and final approval of the manuscript. RD contributed to study design, data acquisition, data analysis, drafting of the manuscript, data interpretation, critical revision for important intellectual content and final approval of the manuscript. DG contributed to study concept and design, data analysis, data interpretation, drafting of the manuscript, critical revision for important intellectual content and final approval of the manuscript. JRC contributed to study design, drafting of the manuscript, critical revision for important intellectual content and final approval of the manuscript. RDK contributed to study design, drafting of the manuscript, critical revision for important intellectual content and final approval of the manuscript. BH contributed to data interpretation, drafting of the manuscript, critical revision for important intellectual content and final approval of the manuscript. SSI contributed to study concept and design, data analysis, data interpretation, drafting of the manuscript, critical revision for important intellectual content and final approval of the manuscript. All authors approved the final manuscript as submitted and agree to be accountable for all aspects of the work.

Funding This study was funded by AbbVie Inc.

Competing interests HL, RD, DG, RDK, BH and SSI are employees of AbbVie and own AbbVie stocks or stock options at the time of the study. JRC has received consulting fees from AbbVie, Amgen, BMS, CORRONA, Janssen, Lilly, Myriad and Pfizer; and research funding from AbbVie, Amgen, BMS, CORRONA, Janssen, Lilly, Myriad and Pfizer.

Patient consent for publication Not required.

Provenance and peer review Not commissioned; externally peer reviewed.

Data availability statement Supplemental data are available in a public, openaccess repository. Data for this study may be obtained from a third party and are not publicly available. No company proprietary data are available. All data relevant to the study are included in the article or uploaded as online supplementary information.

Open access This is an open access article distributed in accordance with the Creative Commons Attribution 4.0 Unported (CC BY 4.0) license, which permits others to copy, redistribute, remix, transform and build upon this work for any purpose, provided the original work is properly cited, a link to the licence is given, and indication of whether changes were made. See: https://creativecommons.org/ licenses/by/4.0/

\section{REFERENCES}

1. Goldhaber SZ. Venous thromboembolism: epidemiology and magnitude of the problem. Best Pract Res Clin Haematol 2012;25:235-42.

2. Zakai NA, McClure LA, Judd SE, et al. Racial and regional differences in venous thromboembolism in the United States in 3 cohorts. Circulation 2014;129:1502-9.

3. Ogdie A, Kay McGill N, Shin DB, et al. Risk of venous thromboembolism in patients with psoriatic arthritis, psoriasis and rheumatoid arthritis: a general population-based cohort study. Euro Heart J 2017

4. Bacani AK, Gabriel SE, Crowson CS, et al. Noncardiac vascular disease in rheumatoid arthritis: increase in venous thromboembolic events? Arthritis Rheum 2012;64:53-61.

5. Kim SC, Schneeweiss S, Liu J, et al. The risk of venous thromboembolism in patients with rheumatoid arthritis. Arthritis Care Res 2013;65:NA-7.

6. Kim SC, Solomon DH, Liu J, et al. Risk of venous thromboembolism in patients with rheumatoid arthritis: initiating disease-modifying antirheumatic drugs. Am J Med 2015;128:539.e7-539.e17.

7. Choi HK, Rho Y-H, Zhu Y, et al. The risk of pulmonary embolism and deep vein thrombosis in rheumatoid arthritis: a UK population-based outpatient cohort study. Ann Rheum Dis 2013;72:1182-7. 
8. Holmqvist ME, Neovius M, Eriksson J, et al. Risk of venous thromboembolism in patients with rheumatoid arthritis and association with disease duration and hospitalization. JAMA 2012;308:1350-6.

9. Bordy R, Totoson P, Prati C, et al. Microvascular endothelial dysfunction in rheumatoid arthritis. Nat Rev Rheumatol 2018;14:404-20.

10. ed)England BR, Thiele GM, Anderson DR, et al. Increased cardiovascular risk in rheumatoid arthritis: mechanisms and implications. In: BMJ. , 2018: 361, k1036. (published Online First: 2018/04/25)

11. Zoller B, Li X, Sundquist J, et al. Autoimmune diseases and venous thromboembolism: a review of the literature. Am J Cardio Dis 2012;2:171-83.

12. American College of Rheumatology updated guideline for the management of rheumatoid arthritis, 2018. Available: https://www. rheumatology.org/Portals/O/Files/Rheumatoid-Arthritis-GuidelineProject-Plan.pdf

13. Jani M, Dixon WG, Kersley-Fleet L, et al. Drug-specific risk and characteristics of lupus and vasculitis-like events in patients with rheumatoid arthritis treated with TNFi: results from BSRBR-RA. RMD Open 2017;3:e000314.

14. Gerhold K, Richter A, Schneider M, et al. Health-Related quality of life in patients with long-standing rheumatoid arthritis in the era of biologics: data from the German biologics register. Rheumatology 2015;54:1858-66.

15. Engbers MJ, van Hylckama Vlieg A, Rosendaal FR. Venous thrombosis in the elderly: incidence, risk factors and risk groups. $J$ Thrombo Haemo 2010;8:2105-12.

16. Benjamin EJ, Virani SS, Callaway CW, et al. Heart disease and stroke statistics - 2018 update: a report from the American Heart Association. Circulation 2018;137:e67-492.

17. Tagalakis $\mathrm{V}$, Kondal $\mathrm{D}$, Ji $\mathrm{Y}$, et al. Men had a higher risk of recurrent venous thromboembolism than women: a large population study. Gend Med 2012;9:33-43.

18. Marshall AL, Bartley AC, Ashrani AA, et al. Sex-based disparities in venous thromboembolism outcomes: National Inpatient Sample (NIS)-based analysis. Vasc Med 2017;22:121-7.

19. Douketis J, Tosetto A, Marcucci M, et al. Risk of recurrence after venous thromboembolism in men and women: patient level metaanalysis. In: BMJ. , 2011: 342, d813.

20. Zöller B, Ji J, Sundquist J, et al. Alcohol use disorders are associated with venous thromboembolism. J Thromb Thrombolysis 2015;40:167-73.

21. Pahor M, Guralnik JM, Havlik RJ, et al. Alcohol consumption and risk of deep venous thrombosis and pulmonary embolism in older persons. J Am Geriatr Soc 1996;44:1030-7.

22. Obesity KC. Cigarette smoking and hypertension were associated with increased risk of pulmonary embolism in women. Evid-Based Cardio Med 1997:1:67.

23. Fahrni J, Husmann M, Gretener SB, et al. Assessing the risk of recurrent venous thromboembolism-a practical approach. Vasc Health Risk Manag 2015;11:451-9.

24. Zhang Y, Yang Y, Chen W, et al. Hypertension associated with venous thromboembolism in patients with newly diagnosed lung cancer. Sci Rep 2016;6:19603.

25. Cushman M. Epidemiology and risk factors for venous thrombosis Semin Hematol 2007:44:62-9.

26. Wattanakit K, Cushman M, Stehman-Breen C, et al. Chronic kidney disease increases risk for venous thromboembolism. J Am Soc Nephrol 2008;19:135-40.

27. Singh G, Rathi AK, Singh K, et al. Venous thromboembolism in cancer patients - magnitude of problem, approach, and management. Indian J Cancer 2017;54:308-12.

28. Cohoon KP, Ashrani AA, Crusan DJ, et al. Is infection an independent risk factor for venous thromboembolism? A population-based, case-control study. Am J Med 2018;131:307-16

29. Bhandari S, Saeian K, Stein D. Increased rate of venous thromboembolism in hospitalized inflammatory bowel disease patients with Clostridium difficile infection. Inflamm Bowel Dis 2018;24:661.

30. Piazza G, Goldhaber SZ, Kroll A, et al. Venous thromboembolism in patients with chronic obstructive pulmonary disease. Am J Med 2012;125:1010-8.
31. Majoor CJ, Bel EHD. Allergic burden and the risk of venous thromboembolism. Eur Res J 2013;42:1158-9.

32. Majoor CJ, Kamphuisen PW, Zwinderman AH, et al. Risk of deep vein thrombosis and pulmonary embolism in asthma. Eur Res $J$ 2013;42:655-61.

33. Galli L, Gerdes VE, Guasti L, et al. Thrombosis associated with viral hepatitis. J Clin Translation Hepatol 2014;2:234-9.

34. Strate LL, Erichsen R, Horváth-Puhó E, et al. Diverticular disease is associated with increased risk of subsequent arterial and venous thromboembolic events. Clin Gastroenterol Hepatol 2014;12:1695-701

35. Kim SY, Hyun JJ, Suh SJ, et al. Risk of vascular thrombotic events following discontinuation of antithrombotics after peptic ulcer bleeding. J Clin Gastroenterol 2016;50:e40-4.

36. Koutroumpakis E, Tsiolakidou G, Koutroubakis I. Risk of venous thromboembolism in patients with inflammatory bowel disease. Semin Thromb Hemost 2013;39:461-8.

37. Lee CW, Liao CH, Lin CL, et al. Depression and risk of venous thromboembolism: a population-based retrospective cohort study. Psychosomatic Medicine 2015;77:591-8.

38. Weinblatt MTP, Burmester GR, Witt S, et al. Cardiovascular safety during treatment with baricitinib in rheumatoid arthritis [abstract] Arthritis Rheumatol 2017;69(suppl 10) https://acrabstracts.org/ abstract/cardiovascular-safety-during-treatment-with-baricitinib-inrheumatoid-arthritis/

39. Scott IC, Hider SL, Scott DL. Thromboembolism with Janus kinase (JAK) inhibitors for rheumatoid arthritis: how real is the risk? Drug Safety 2018;41:645-53.

40. Desai RJ, Pawar A, Weinblatt ME, et al. Comparative risk of venous thromboembolism in rheumatoid arthritis patients receiving tofacitinib versus those receiving tumor necrosis factor inhibitors: an observational cohort study. Arthritis Rheumatol 2019;71:892-900.

41. Tagalakis V, Kahn SR. Determining the test characteristics of claims-based diagnostic codes for the diagnosis of venous thromboembolism in a medical service claims database. Pharmacoepidemiol Drug Saf 2011;20:304-7.

42. Tamariz L, Harkins T, Nair V. A systematic review of validated methods for identifying venous thromboembolism using administrative and claims data. Pharmacoepidemiol Drug Saf 2012;21:154-62

43. Yang D, Jarrod E. A unified approach to measuring the effect size between two groups using SAS $尺$. SAS global forum 2012.

44. Li P, Blum MA, Von Feldt J, et al. Adherence, discontinuation, and switching of biologic therapies in Medicaid enrollees with rheumatoid arthritis. Value in Health 2010;13:805-12.

45. Bernatsky S, Ehrmann Feldman D. Discontinuation of methotrexate therapy in older patients with newly diagnosed rheumatoid arthritis: analysis of administrative health databases in Quebec, Canada. Drugs \& Aging 2008;25:879-84.

46. Nelson WB. Analysis of complete data: Poisson data. In: Applied life data analysis. Schenectady, New Jersey: John Wiley \& Sons, Inc, 1982: 201.

47. Annual estimates of the resident population for selected age groups by sex for the United States, states, counties, and Puerto Rico Commonwealth and Municipios: April 1, 2010 to July 1, 2015. 2015 population estimates. United States Census Bureau. American Fact Finder. Available: https://factfinder.census.gov/faces/tableservices/ jsf/pages/productview.xhtml?src=bkmk

48. HKT N, Filardo G, Zheng G. Confidence interval estimating procedures for standardized incidence rates. Computational Statistics \& Data Analysis 2008:52:3501-16.

49. Desai RJ, Gagne JJ, Lii J, et al. Comparative risk of incident venous thromboembolism in patients with inflammatory bowel disease initiating tumour necrosis factor- $\alpha$ inhibitors or nonbiologic agents: a cohort study. Can Med Assoc J 2017;189:E1438-E1447.

50. Branchford BR, Carpenter SL. The role of inflammation in venous thromboembolism. Frontiers in Pediatrics 2018;6.

51. White RH, Garcia M, Sadeghi B, et al. Evaluation of the predictive value of ICD-9-CM coded administrative data for venous thromboembolism in the United States. Thromb Res 2010;126:61-7.

52. Roy P-M, Moumneh T, Penaloza A, et al. Outpatient management of pulmonary embolism. Thromb Res 2017;155:92-100.

53. Harrell FE. Regression modeling strategies: with applications to linear models, logistic regression and survival analysis. New York: Springer, 2001. 